\title{
Identification of Differentially Expressed Genes and Key Pathways in the Dorsal Root Ganglion After Chronic Compression
}

\author{
Zhanhui Du ${ }^{1,2}$, Sen Yin ${ }^{3}$, Xiuhui Song ${ }^{4}$, Lechi Zhang ${ }^{5}$, Shouwei Yue ${ }^{1}$, Xiaofeng Jia ${ }^{6,7,8}$ \\ and Yang Zhang ${ }^{1,6 *}$
}

${ }^{1}$ Department of Physical Medicine \& Rehabilitation, Qilu Hospital, Shandong University, Jinan, China, ${ }^{2}$ Heart Center, Qingdao Women and Children's Hospital, Qingdao, China, ${ }^{3}$ Department of Neurology, Qilu Hospital, Shandong University, Jinan, China, ${ }^{4}$ Department of Neurosurgery, The People's Hospital of Jimo City, Qingdao, China, ${ }^{5}$ Department of Physical Medicine \& Rehabilitation, Suzhou Hospital Affiliated to Nanjing Medical University, Suzhou, China, ${ }^{6}$ Department of Neurosurgery, University of Maryland School of Medicine, Baltimore, MD, United States, ${ }^{7}$ Department of Orthopaedics, Anatomy \& Neurobiology, University of Maryland School of Medicine, Baltimore, MD, United States, ${ }^{8}$ Departments of Biomedical Engineering, Anesthesiology and Critical Care Medicine, The Johns Hopkins University School of Medicine, Baltimore, MD, United States

\section{OPEN ACCESS}

Edited by: Aurelio Galli,

University of Alabama at Birmingham, United States

Reviewed by: Heinrich J. G. Matthies, University of Alabama at Birmingham, United States

Kai K. Kummer, Innsbruck Medical University, Austria

*Correspondence: Yang Zhang zhangyang982003@163.com

Received: 23 January 2020 Accepted: 14 April 2020

Published: 05 May 2020

Citation:

Du Z, Yin S, Song X, Zhang L, Yue S, Jia $X$ and Zhang $Y$ (2020) Identification of Differentially Expressed Genes and Key Pathways in the Dorsal Root Ganglion After Chronic Compression.

Front. Mol. Neurosci. 13:71. doi: 10.3389/fnmol.2020.00071
Neuropathic pain (NP) is caused by primary or secondary impairment of the peripheral or central nervous systems. Its etiology is complex and involves abnormal patterns of gene expression and pathway activation. Using bioinformatics analysis, we aimed to identify NP-associated changes in genes and pathways in L4 and L5 dorsal root ganglia (DRG) in a rat model of NP induced by chronic compression of the DRG (CCD). Genome-wide transcriptional analyses were used to elucidate the molecular mechanisms underlying NP. We screened differentially expressed genes (DEGs) 7 days after CCD in comparison with sham-operated controls. Quantitative real-time polymerase chain reaction (RTqPCR) and western blotting were used to confirm the presence of key DEGs. Kyoto Encyclopedia of Genes and Genomes (KEGG)-pathway analysis of DEGs and global signal transduction network analysis of DEGs were also conducted. The CCD group developed clear mechanical and thermal allodynia in the ipsilateral hind paw compared with the sham group. This comparison identified 1,887 DEGs, with 1156 upregulated and 731 downregulated DEGs, and 123 DEG-enriched pathways. We identified the key candidate genes that might play a role in the development of NP, namely syndecan 1 (Sdc1), phosphatidylinositol-4,5-bisphosphate 3-kinase, catalytic subunit gamma (Pi3k), Janus kinase 2 (Jak2), jun proto-oncogene, AP-1 transcription factor subunit (Jun), and interleukin $6(\mathrm{IL}-6)$ by analyzing the global signal transduction network. RT-qPCR and western blot analysis confirmed the microarray results. The DEGs Sdc1, Pi3k, Jak2, Jun, and $I L-6$, and the cytokine signaling pathway, the neuroactive ligand-receptor interaction, the toll-like receptor signaling pathway, and the PI3K-Akt signaling pathway may have decisive modulatory roles in both nerve regeneration and NP. These results provide deeper insight into the mechanism underlying NP and promising therapeutic targets for its treatment.

Keywords: neuropathic pain, bioinformatic analysis, differentially expressed gene, gene ontology analysis, pathway analysis, KEGG database, CCD 


\section{INTRODUCTION}

Neuropathic pain (NP; or spontaneous pain) lacks organic causes and usually arises from a primary lesion or dysfunction of the nervous system (Woolf and Mannion, 1999). It has a global incidence of 7-10\% (van Hecke et al., 2014). Patients present with varying degrees of mechanical and heat hyperalgesia and sleep and anxiety disorders, which impair their quality of life (Attal et al., 2011; Finnerup et al., 2016). The efficacy of pharmacotherapy for treating NP is limited, owing to undesirable side effects and poor responsiveness (Jacobs, 2017). Although several novel therapies have been developed for NP, the mechanism underlying NP remains unclear. Clarifying the molecular mechanism underlying NP remains an unresolved issue in the field of medical research, and a solution could lead to better clinical management of NP.

Neuronal sensitization is thought to play a key role in the development and maintenance of NP (Teixeira et al., 2015). Inflammation is inevitable during this process, and allodynia and hyperalgesia are common features. Gene expression is altered in the spinal cord and dorsal root ganglion (DRG), as evidenced by inconsistent results in different animal models of peripheral nerve injury (Wang et al., 2017; Liu et al., 2018; Zhao et al., 2020). The chronic compression of the DRG (CCD) model mimics clinical radicular pain, such as spontaneous pain, hyperalgesia, and allodynia. In comparison with other models, there are distinct properties of the CCD model, such as maintenance of peripheral input, absence of motor disorders, and ectopic discharge of neurons. Studies on CCD are essential to improve understanding of comprehensive neuropathic pain; they will also provide an opportunity to compare the results with other models.

The DRG has been proven to be involved in NP and nerve regeneration as a modulatory locus, and it may be the most important therapeutic target. Several studies have reported that the expression of ion channels, chemokines, and receptors changed after CCD (Oh et al., 2001). However, these studies focused on individual genes and failed to provide insight into the overall changes and the networks between the target genes. New insights have been achieved into the diagnosis and treatment of several diseases, with the development of high-throughput gene screening technology such as microarrays. The use of microarrays has rendered gene expression profiling a robust and straightforward process for studying the molecular features of diseases at the systemic level. Therefore, research on the molecular mechanism of NP based on the CCD model using microarrays may be useful for the development of appropriate clinical therapies for NP. This study aimed to explore the central genes that are dysregulated and the signaling pathways that are activated in a rat CCD model of NP using bioinformatics analysis.

\section{MATERIALS AND METHODS}

\section{Experimental Animals}

A total of 34 Wistar rats (adult male, weighing 180-200 g) were purchased from the Experimental Animal Center of Shandong
University and housed in a room with pathogen-free air at $20 \pm 2{ }^{\circ} \mathrm{C}$, with two rats in a cage, at a 12 -h light/dark cycle. Water and food were available ad libitum. The experimental operation began on the 8th day so that the animals were habituated to their environment. The rats were divided into the sham and CCD groups randomly, in which 18 rats were used for microarray analysis (eight in the CCD group; 10 in the sham group) and 16 rats were used for behavioral studies and molecular experiments (eight in each group). Each behavioral study began $10 \mathrm{~min}$ after the animal entered the experimental environment. All experimental procedures were approved by the Animal Care and Use Committee of Shandong University.

\section{CCD Model}

CCD was induced as described earlier (Jia et al., 2018). In summary, after the rats were anesthetized, the paraspinal muscles were separated to unilaterally expose the transverse processes and intervertebral foramina of L4 and L5. Two L-shaped stainless steel rods (diameter $=0.63 \mathrm{~mm}$ and length $=4 \mathrm{~mm}$ ) were implanted into the intervertebral foramina of L4 and L5 at an oblique angle of $30^{\circ}$ with the spinal column to compress the DRGs. The other end of the bar was maintained out of the intervertebral foramen. Rats in the sham-operated group underwent the same procedure without steel rod insertion. Those exhibiting autophagy, tactile sensory deficiencies, or disabilities were excluded. The rats were administered deep anesthesia with isoflurane and were sacrificed after behavioral testing on the 7th-day post-surgery. The L4 and L5 DRG were then harvested for microarray analysis and validation tests.

\section{Nociceptive Behavioral Testing}

Mechanical hypersensitivity was determined by the same experimenter before the procedure and on the 1st, 3rd, 5th, and 7th days after CCD induction, respectively (Qu et al., 2016). The paw withdrawal threshold was automatically recorded by the BEM-404 mechanical analgesia tester [Chinese Academy of Medical Sciences (CAMS), Beijing, China] and the cutoff force was set at $50 \mathrm{~g}$. Tests were started $10 \mathrm{~min}$ after the rats entered the test environment. A rigid tip was applied perpendicularly to the mid-plantar surface of the hind paw. Brisk withdrawal or paw flinching was considered a positive response. The number displayed on the digital monitor was recorded as the paw withdrawal mechanical threshold (PWMT). Three successive stimuli were applied. The mean of three successive measurements was recorded as the PWMT of each animal.

Thermal paw withdrawal latency (TPWL) was measured using the BME-410C thermal analgesia tester (CAMS) as described previously (Jia et al., 2018). The light source was a $12 \mathrm{~V} / 10 \mathrm{~W}$ halogen lamp and the thermal intensity was set at $60^{\circ} \mathrm{C}$. Radiant light was focused on the center of the hind paw at about $1 \mathrm{~cm}$, and TPWL was recorded when the rat lifted or licked the hind paw. A 25-s cutoff period was used to avoid tissue damage. The test was repeated three times for each rat with a 5-min interval between successive measurements; the mean of three successive measurements was recorded as the TPWL of each animal. 


\section{Total RNA Extraction and Microarray Assay} One sample for each group (sham and CCD) was collected by pooling the L4 and L5 DRG of two rats. Total RNA was extracted and purified with an RNeasy Mini Kit (Qiagen, Hilden, Germany) according to the manufacturer's instructions. The quality and amount of RNA were measured using NanoDrop 2000 (Thermo, USA). mRNA expression profiling was performed using Affymetrix Clariom D Rat array (Affymetrix; Thermo Fisher Scientific Inc., Waltham, MA, USA), which contains 46,800 gene-level probe sets. GeneChips were washed, stained, and subsequently scanned using the $30007 \mathrm{G}$ GeneChip Scanner. Raw data were analyzed using the Robust Multichip Analysis (RMA) algorithm with the Affymetrix Expression Console Software (version 1.2.1). The values were presented as $\log _{2}$ RMA signal intensities. The microarray data discussed in this article have been submitted to the NCBI Gene Expression Omnibus and can be accessed through the GSE accession number (GSE145222).

\section{Data Analysis}

Differentially expressed genes (DEGs) were identified using the random variance model $t$-test and false discovery rate (FDR) analysis for comparisons between two groups. DEGs were considered to be present if $p<0.05$ and the absolute value of fold change $(\mathrm{FC})>2$. DEGs were considered up or down-regulated if there was at least a 2 -fold change in the positive or negative direction, respectively. Hierarchical clustering was performed using Cluster Treeview software (Palo Alto, CA, USA) to observe the DEG-expression pattern.

Gene ontology (GO) analysis was used to classify DEGs into different hierarchical categories based on the biological process and molecular function and reveal the gene regulatory network. Pathway analyses of DEGs were determined according to the Kyoto Encyclopedia of Genes and Genomes (KEGG) database. The significance of the pathway was determined using Fisher's exact test. The $P$-value was corrected by FDR analysis and a $p$ value $<0.05$ was considered statistically significant.

A global signal transduction network was constructed using Cytoscape V3.6.0 based on the KEGG database to illustrate the inter-gene signaling of DEGs and discover the core genes playing an important role in this network. The nodes and lines in the network graphs represented the genes and the interactions between the nodes, respectively, such as inhibition of phosphorylation. "In-degree," "out-degree," or "degree" represented the number of links of a single node with its upstream genes, downstream genes, or all binding genes, respectively. The hub gene was selected based on the degrees. Genes with higher degrees had a more crucial position in the network.

\section{Real-Time Polymerase Chain Reaction Analysis}

Quantitative real-time polymerase chain reaction (RT-qPCR) was performed as described previously (Du et al., 2018). The total RNA was extracted and reverse-transcribed into cDNA. qPCR was performed on an iQ5 Real-Time PCR cycler (Bio-Rad,
Hercules, CA, USA). All samples were normalized to Gapdh values. Data are shown as fold changes $\left(2^{-\Delta \Delta C T}\right)$. The primer sequence used is shown in Table $\mathbf{1 .}$

\section{Western Blotting}

Total proteins were extracted from tissues using RIPA Lysis Buffer (Beyotime, Haimen, Jiangsu, China). Equal amounts of the protein were separated using sodium dodecyl sulfatepolyacrylamide gel electrophoresis and transferred to a polyvinylidene fluoride membrane, followed by overnight incubation with primary antibodies at $4{ }^{\circ} \mathrm{C}$. Primary antibodies against the following proteins were used: Pi3k (1:300, 20584-1AP, Proteintech), Jak2 (1:4,000, ab108596, Abcam), Gng7 (1:800, GTX65584, GeneTex), Jun (1:1,500, 66313-1-Ig, Proteintech), IL-6 (1:1,000, ab9324, Abcam), Met (1:1,500, ab51067, Abcam), Pdgfra (1:1,000, ab203491, Abcam), Camk2b (1:1,000, ab34703, Abcam), Ptgs2 (1:1,000, ab15191, Abcam), Sdc1 (1:1,000, ab128936, Abcam), and Gapdh (1:10,000; SAB2100894; Sigma-Aldrich; Merck KGaA). Target protein expression was normalized to Gapdh expression.

\section{Statistical Analysis}

All data are presented as mean \pm SEM. The repeated measures two-way ANOVA test was used for the statistical analysis of the sham and CCD groups using SPSS 20.0 software (IBM Company, Armonk, NY, USA). Significant GO categories and pathways were selected based on Fisher's exact test, and all $p$-values

TABLE 1 | Primers used in Quantitative real-time polymerase chain reaction (RT-PCR) analysis.

\begin{tabular}{|c|c|}
\hline Gene & Sequence $5^{\prime}-3^{\prime}$ \\
\hline \multirow[t]{2}{*}{ Pi3k } & F: TCTCTGGACCTGTGCCTTCT \\
\hline & R: GCCTGTCACCTATCCCAAGA \\
\hline \multirow[t]{2}{*}{ Jak2 } & F: AGATATGCAAGGGCATGGAG \\
\hline & R: GTCAAGGATTCGGGAGCATA \\
\hline \multirow[t]{2}{*}{ Gng7 } & F: ACTTTGTCTCCGAAGCCTGA \\
\hline & R: AGAAAATGGCCACAGTCCAC \\
\hline \multirow[t]{2}{*}{ Jun } & F: CAGGTGGCACAGCTTAAACA \\
\hline & R: CGCAACCAGTCAAGTTCTCA \\
\hline \multirow[t]{2}{*}{ IL6 } & F: CCGGAGAGGAGACTTCACAG \\
\hline & R: ACAGTGCATCATCGCTGTTC \\
\hline \multirow[t]{2}{*}{ Met } & F: CAGCGGCAATTCTAGACACA \\
\hline & R:CTGAAGCTGCTTGTCACTCG \\
\hline \multirow[t]{2}{*}{ Pdgfra } & F: CACAATAACGGGAGGCTGGT \\
\hline & R: GTTCTGACGTGGCTTTCAAGG \\
\hline \multirow[t]{2}{*}{ Camk2b } & F: GGAGTCAAGCCCCAGACAAAC \\
\hline & R: GTGTTGGTGCTGTCGGAAGATT \\
\hline \multirow[t]{2}{*}{ Ptgs2 } & F: GTGGGATGACGAGCGACTG \\
\hline & R: CCGTGTTCAAGGAGGATGG \\
\hline \multirow[t]{2}{*}{ Sdc1 } & F: ACCATCAGCCTCCAAGTGTG \\
\hline & R: TGAAGTCTTGTTCTCCAGAGCC \\
\hline \multirow[t]{2}{*}{ Gapdh } & F: AGGTCGGAGTCA ACGGATTTGGT \\
\hline & R: CATGTGGGCCATGAGGTCCACCAC \\
\hline
\end{tabular}

Pi3k, phosphatidylinositol-4,5-bisphosphate 3-kinase catalytic subunit gamma; Jak2, Janus kinase 2; Gng7, G protein subunit gamma 7; Jun, Jun proto-oncogene, AP-1 transcription factor subunit; IL6, interleukin 6; Met, MET proto-oncogene, receptor tyrosine kinase; Pdgfra, platelet-derived growth factor receptor alpha; Camk2b, calcium/calmodulin-dependent protein kinase Il beta; Ptgs2, prostaglandin-endoperoxide synthase 2; $S d c 1$, syndecan 1; $F$, forward; $R$, reverse. 
were FDR adjusted $p$-values. A $p$-value $<0.05$ was considered statistically significant.

\section{RESULTS}

\section{PWMT and TPWL Changes After CCD Operation}

The CCD group developed clear mechanical allodynia in the ipsilateral hind paw, compared with the sham group (Figure 1A). The PWMT decreased significantly 3rd $(p=0.008)$, 5th $(p=0.002)$, and 7 th $(p=0.001)$ days after CCD operation $(n=8$ in each group).

Thermal hyperalgesia was determined using the TPWL test. As indicated in Figure 1B, the TPWL decreased significantly (similar to the PWMT) from the 1st to 7th-day post-surgery (all $p \leq 0.001)$, when compared to the sham-operated rats $(n=8$ in each group).
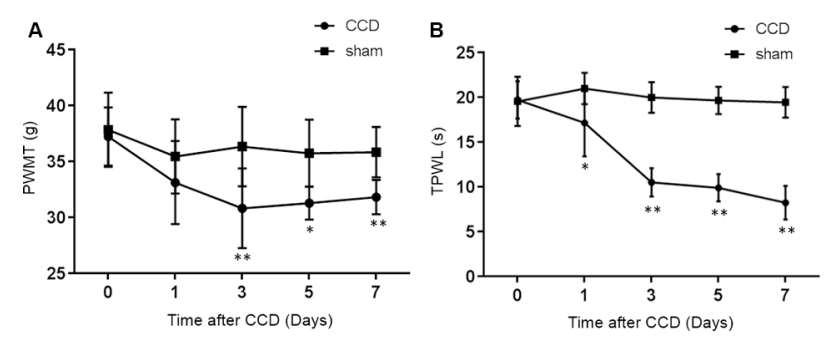

FIGURE 1 | PWMT and TPWL changes after chronic compression of the DRG (CCD) operation. (A) The PWMT decreased significantly 3rd, 5th, and 7th days after CCD surgery when compared with the sham group. (B) Compared to the sham group, TPWL decreased from the 1st to 7th-day post-surgery; $n=8$ in both groups. PWMT, the paw withdrawal mechanical threshold; TPWL, thermal paw withdrawal latency. ${ }^{*} P<0.05$ and ${ }^{* *} P<0.01$ compared with the sham group.

\section{Analysis of mRNA With Differential Expression}

Microarray analysis identified 1,887 differentially expressed genes (DEGs) in comparing the CCD and sham groups. A volcano plot was applied to visualize the genes identified (Figure 2A). Among all these genes, 1,156 were upregulated and 731 were downregulated (Figure 2B). Figure 2C shows a heatmap of DEG expression; it demonstrates that the mRNA expression profiles of the two groups were distinct ( $n=4$ in the CCD group, $n=5$ in the sham group).

\section{GO-Term Enrichment Analysis of DEGs}

GO analysis was performed to explore the main cell functions of DEGs. The top five GO terms among the CCD-upregulated genes (upGOs) were as follows: response to lipopolysaccharide, inflammatory response, cellular response to lipopolysaccharide, integrin-mediated signaling pathway, and chemotaxis (Figure 3A). The GO terms for CCD-downregulated genes (downGOs) included regulation of ionic transmembrane transport, potassium ion transmembrane transport, regulation of potassium transmembrane transport, chemical synaptic transmission, and positive regulation of synapse assembly (Figure 3B).

\section{KEGG-Pathway Analysis of DEGs}

The DEGs were subjected to pathway analysis based on the KEGG database. The analysis revealed 123 enriched pathways for both upregulated and downregulated DEGs. As shown in Figure 4, several important pathways were altered in our NP model, including cytokine-cytokine receptor interaction, neuroactive ligand-receptor interaction, cell adhesion molecules, toll-like receptor signaling pathway, phagosome, lysosome, PI3K-Akt signaling pathway, cell cycle, TNF signaling pathway, Jak-STAT signaling pathway, and apoptosis.
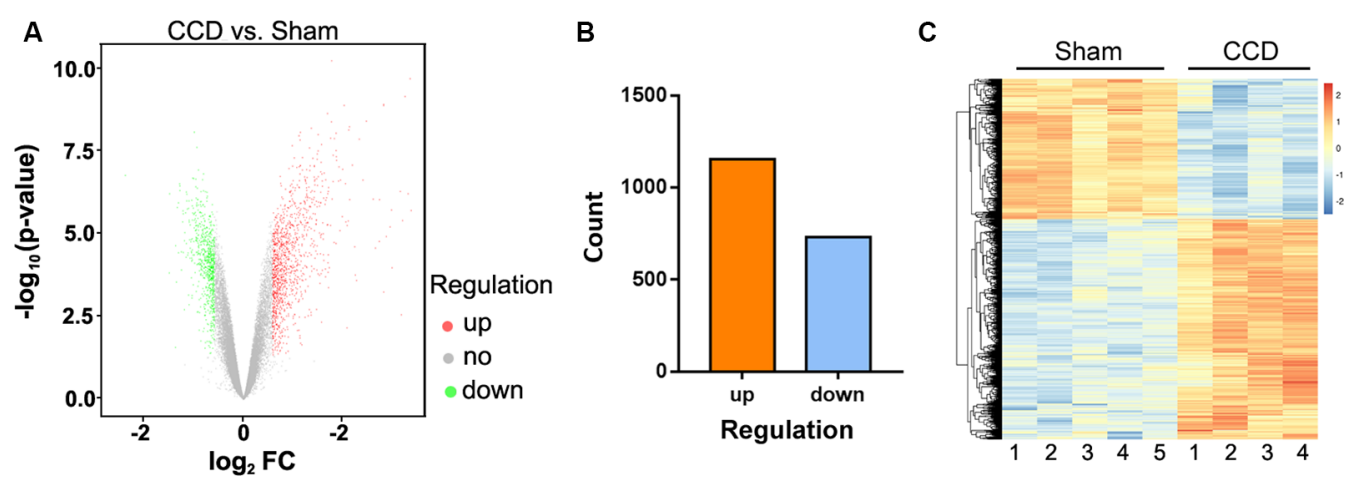

FIGURE 2 | DEGs between the CCD group and the sham group. (A) Volcano plot of the $-\log _{10}(\boldsymbol{D}$-value) against the log 2 FC of each gene. Red dot indicates an upregulated DEG, whereas green dot indicates a downregulated gene. Gray indicates that there is no statistically significant difference in gene expression. (B) There were 1,887 DEGs identified between the CCD group and the sham group. Of these genes, 1,156 were upregulated and 731 were downregulated. (C) Hierarchical clustering shows the DEGs between the two groups. The number at the bottom represents the name of the samples. The color scale at the top right represents the normalized expression data, which represents the degree of gene clustering. The upregulated genes are indicated in red, and the downregulated genes are indicated in blue. DEGs, differentially expressed genes; CCD, chronic compression of the dorsal root ganglion surgery group; FC, fold change; sham, compression of the dorsal root ganglion sham group. The $p$-value was corrected by false discovery rate (FDR). 


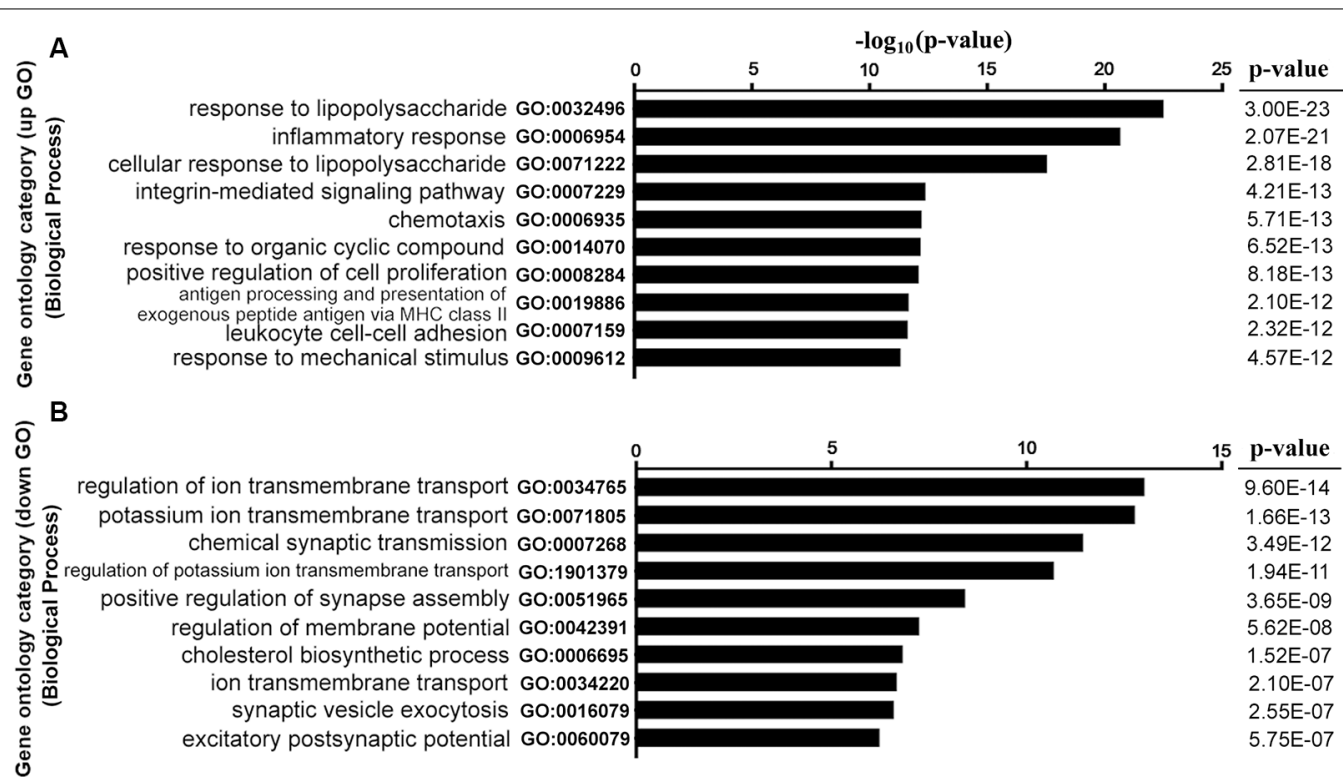

FIGURE 3 | Functional enrichment analysis for DEGs. GO terms enriched by upregulated (upGOs) and downregulated genes (downGOs) are shown as (A) and (B), respectively. The horizontal axis represents the value of $-\log _{10}(p$-value), and the vertical axis represents the category of $\mathrm{GO}$ biological processes. $P$-value was adjusted by FDR. GO, gene ontology. BP, biological process.

\section{Functional Gene Pathway Enrichment}

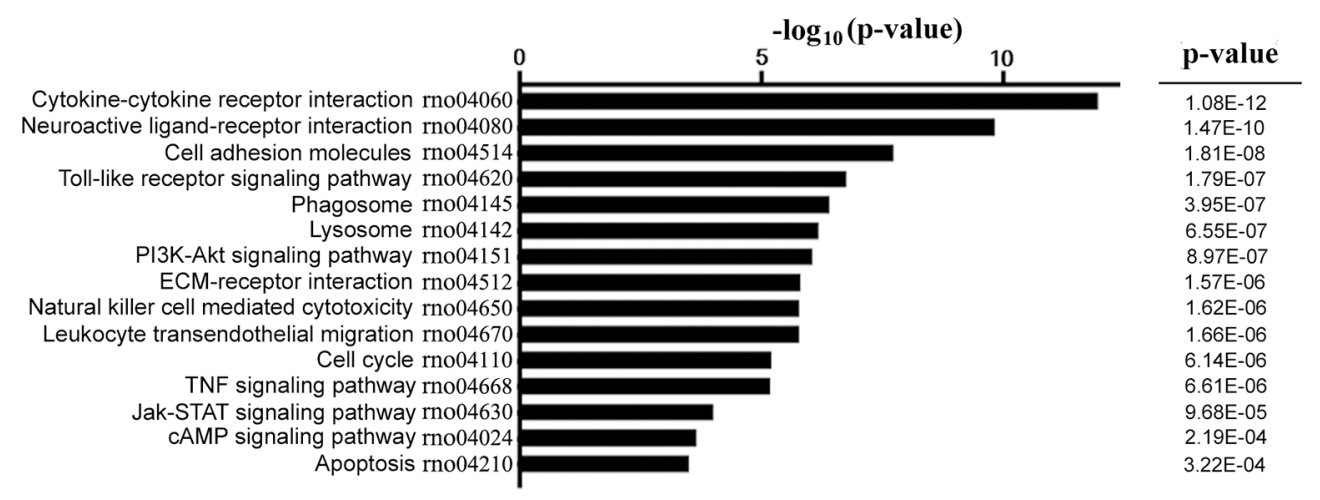

FIGURE 4 | KEGG pathway enrichment analysis for DEGs. The vertical axis on the left side represents the name of the pathway, and the horizontal axis at the top represents the value of $-\log _{10}(p$-value). P-value was adjusted by FDR. KEGG, Kyoto Encyclopedia of Genes and Genomes.

\section{Global Signal Transduction Network Analysis of DEGs}

The candidate genes that could play a key role in the development of NP were screened using global signal transduction network analysis. As illustrated in Figure 5, the high-degree genes were: phosphatidylinositol-4,5-bisphosphate 3-kinase, catalytic subunit gamma (Pi3k), Janus kinase 2 (Jak2), G protein subunit gamma 7 (Gng7), Jun proto-oncogene aka AP-1 transcription factor subunit (Jun), interleukin 6 (IL-6), MET proto-oncogene aka receptor tyrosine kinase (Met), platelet-derived growth factor receptor alpha (Pdgfa), calcium/calmodulin-dependent protein kinase II beta (Camk2b), prostaglandin-endoperoxide synthase 2 (Ptgs2), and syndecan 1 (Sdc1). Gng7 and Camk2b were downregulated while the others were upregulated (Table 2).

qPCR was performed to validate the mRNA levels of the ten DEGs. Gng7 and Camk2b were found to be downregulated and the remaining genes were upregulated (Figure 6A), supporting the microarray results. Moreover, western blotting confirmed that the protein levels of Pi3k, Jak2, Jun, IL-6, and Sdc1 were markedly higher in the CCD group than in the sham group (Figure 6B). However, there was no difference in protein expression of Gng7, Met, Pdgfra, Camk2b, and Ptgs2 (data are not shown in the figure). 


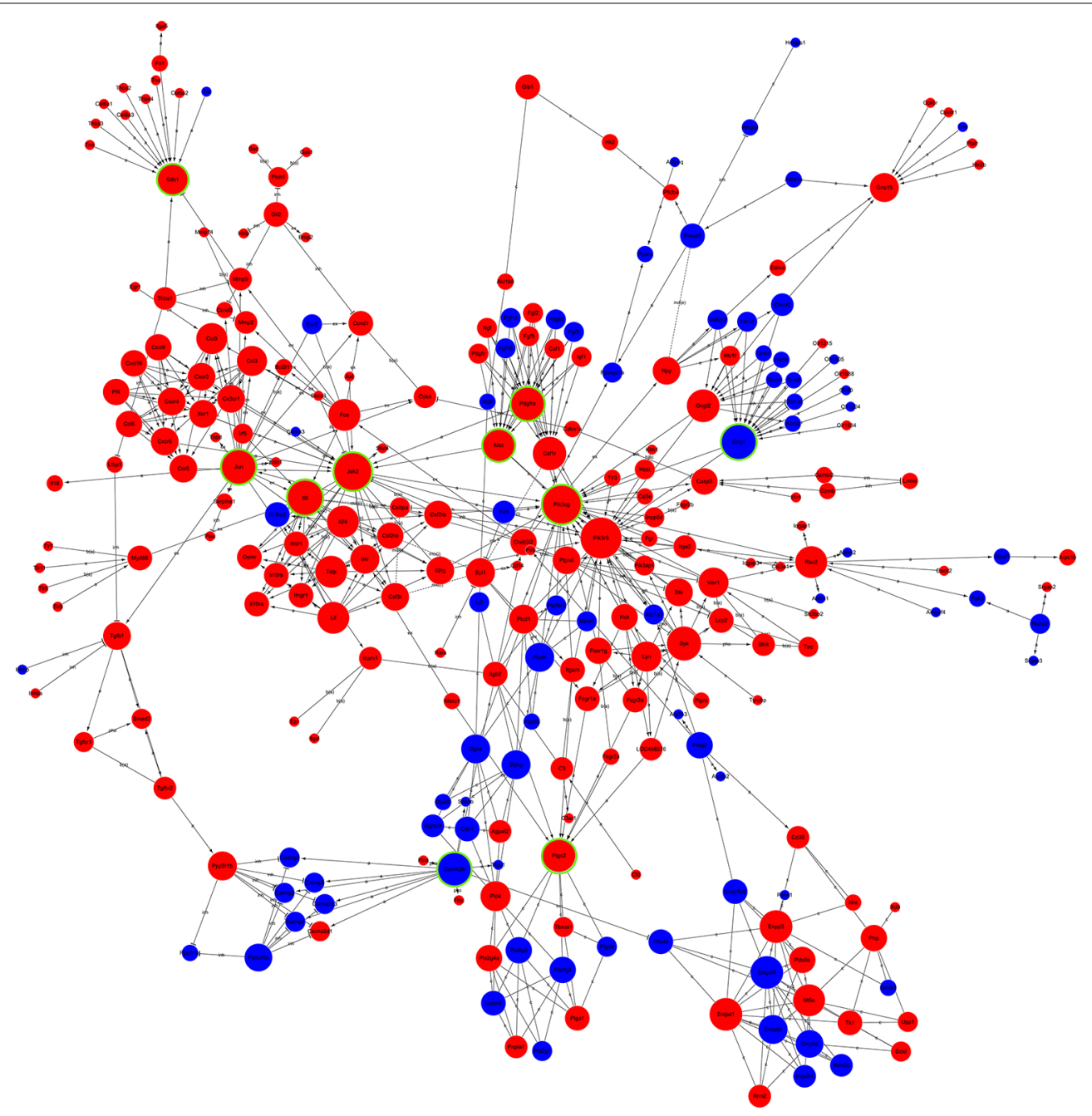

FIGURE 5 | Global signal transduction network of DEGs. The red node represents an upregulated gene, and the blue node represents the downregulated gene. The lines exhibit the interaction between the genes. The size of the node indicates the degree of interacting with other genes. The more important the genes are, the larger the node is. Nodes with green rings were the genes that were selected to be experimentally validated.

TABLE 2 | Ten key genes identified by global signal transduction network analysis.

\begin{tabular}{|c|c|c|c|c|c|c|c|}
\hline Gene symbol & Offical full name & Style & Degree & Indegree & Outdegree & $p$-value & Fold change \\
\hline Pi3k & phosphatidylinositol-4,5-bisphosphate 3-kinase catalytic subunit gamma & up & 30 & 24 & 6 & 0.003 & 1.79 \\
\hline Jak2 & Janus kinase 2 & up & 26 & 23 & 3 & 0.0008 & 1.75 \\
\hline Gng7 & G protein subunit gamma 7 & down & 18 & 14 & 4 & 0.002 & -1.65 \\
\hline Jun & Jun proto-oncogene, AP-1 transcription factor subunit & up & 18 & 2 & 16 & 0.0005 & 1.89 \\
\hline IL6 & interleukin 6 & up & 16 & 5 & 11 & 0.001 & 2.24 \\
\hline Met & MET proto-oncogene, receptor tyrosine kinase & up & 13 & 10 & 3 & 7.321E-0.5 & 2.34 \\
\hline Pdgfra & platelet derived growth factor receptor alpha & up & 13 & 10 & 3 & 0.0006 & 1.65 \\
\hline Camk2b & calcium/calmodulin-dependent protein kinase II beta & down & 12 & 0 & 12 & 0.0006 & -1.51 \\
\hline Ptgs2 & prostaglandin-endoperoxide synthase 2 & up & 12 & 10 & 2 & 0.014 & 2.51 \\
\hline Sdc1 & syndecan 1 & up & 12 & 12 & 0 & 8.327E-0.5 & 1.96 \\
\hline
\end{tabular}

$P$-value was corrected by FDR.

\section{DISCUSSION}

$\mathrm{NP}$ is a commonly encountered neurological condition wherein patients feel pain in the absence of external stimuli; this is termed "spontaneous pain." Alterations in gene and protein expression characterize the development and maintenance of NP (Gold and Gebhart, 2010; Wang et al., 2017). In the present study, we analyzed the mRNA expression patterns after CCD 


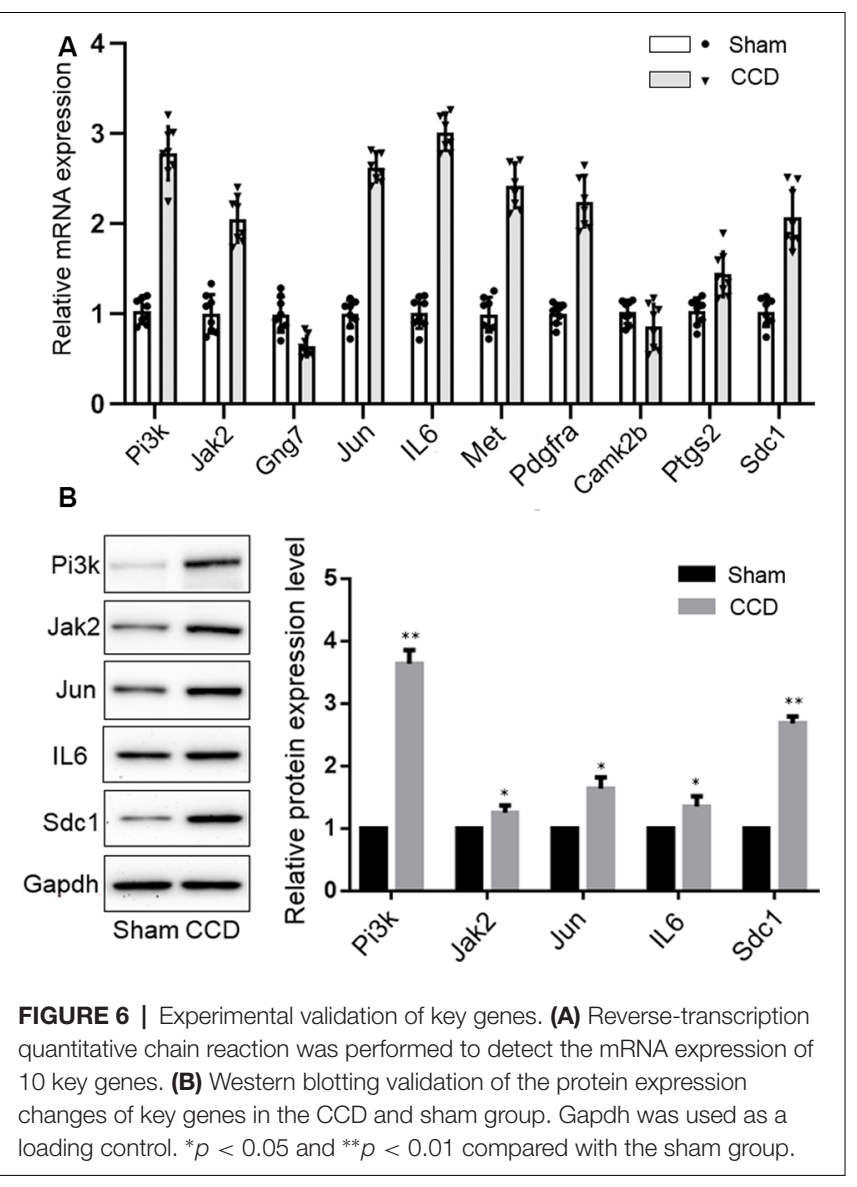

in the L4 and L5 DRG, which are important sites of pain regulation, synaptic plasticity, and NP treatment. Comparisons of sham-operated and CCD rats revealed expression changes in 1,887 genes. GO term enrichment analysis-term enrichment analysis of DEGs showed that the top GO terms among upGOs included a response to inflammation, integrin-mediated signaling pathway, and chemotaxis; those among downGOs included regulation of ionic transmembrane transport.

Recent bioinformatic analysis has explored the genetic processes and molecular mechanisms underlying NP, and several DEGs have been identified (Wang et al., 2017; Liu et al., 2018; Yang et al., 2018; Tang et al., 2020). Cobos et al. (2018) obtained a PPI network consisting of 31 nodes and 442 edges, which revealed certain key signaling molecules such as ATF3, JUN, BDNF, and MAPK1/3 in the sciatic nerve injury (SNI) model. Using the same dataset, Tang et al. (2020) identified additional 10 major DEGs, such as Npy, Atf3, and Gpr151, that were predominantly associated with cytokine-cytokine receptor interactions and the p53 signaling pathway. CCL2, NF-KB1, and C1Q in the dorsal horns (Wang et al., 2017), p53 and active caspase-3 in the DRG (Gao et al., 2018), Ccl3, Atf3, and Tgifl in the spinal cord (Zhang and Yang, 2017) were separately considered to contribute to the generation of NP after SNI, chronic constriction injury (CCI), and spinal cord injury (SCI). Our study showed that Jun and IL-6 may evoke the progression of NP after CCD; this was consistent with other bioinformatics analysis (Liu et al., 2018; Yang et al., 2018; Tang et al., 2020). Therefore, the genetic targets differed with the pathological conditions and analytical methods. The CCD model mimics the effects of protrusion of an intervertebral disc through the injuring of both sensory neurons and spinal nerves; this distinguishes it different from other animal models. Moreover, the steel rods used in the protocol could potentially induce sterile inflammation and damage spinal segmental vessels passing through the intervertebral foramina. Hence, spinal compression and the accompanying inflammation may contribute to the behavioral and physiological consequences of CCD and greater complexity in the local microenvironments (Yang et al., 2018). This is consistent with our observation that the upGO terms enriched by DEGs after CCD were the inflammatory response and integrin-mediated signaling pathway, that were associated with mechanotransduction.

Meanwhile, the main downGOs categories of DEGs were the regulation of ion transmembrane transport, potassium ion transmembrane transport, and regulation of membrane potential. It is well known that potassium ion channels, inward rectifying potassium channels (Kir) in particular, maintain strongly negative resting membrane potential (Rosenhouse-Dantsker, 2019). Thus, downGOs of potassium ion transmembrane transport and regulation of membrane potential may be associated with neuronal somal hypeand peripheral axotomyrexcitability and spontaneous action potentials after CCD. However, Gao et al. (2018) reported that the DEGs associated with the regulation of transmembrane transport and regulation of membrane potential were upregulated after CCI. This further demonstrates the importance of research in different pathological conditions. Interestingly, the downGOs included the cholesterol biosynthetic process. It has been demonstrated that many ion channels including Kir channels, in particular, are modulated by cholesterol (Rosenhouse-Dantsker, 2019). Therefore, our findings provide a new insight into the understanding of the mechanism of cellular excitability and ion channel rearrangements after CCD.

We found that the expression of $S d c 1$ was significantly increased 7 days after CCD. Sdc1 is expressed in C-fiber sensory neurons distributed in peripheral and central axons. It was demonstrated that $S d c 1$ could reduce neurite extension in primary cultured DRG neurons in both, the proximal region and distal regions of the injured sciatic nerve (Murakami et al., 2015). Sciatic nerve axotomy and infraorbital nerve axotomy induced $S d c 1$ in primary sensory neurons, which may contribute to the development of abnormal nociception (Couchman, 2003). Moreover, Sdc1 expression plays a functional role in nerve regeneration and synaptic plasticity in injured primary sensory neurons (Edwards and Hammarlund, 2014; Bray et al., 2019). The increased expression of $S d c 1$ may have multiple functions in nerve regeneration and the development of abnormal nociception after CCD; this needs further research.

As shown in Figure 5, the expression of Jun and IL-6 increased significantly 7 days after CCD; this was consistent with findings from previous studies (Ding et al., 2016; Zhao et al., 2020). Jun is expressed at lower levels in normal nervous 
systems; however, it is upregulated in response to harmful stimuli such as dorsal column transection, root rhizotomy, and peripheral axotomy, et cetera (Kenney and Kocsis, 1998; Li et al., 2013). Notably, the c-Jun N-terminal kinase (JNK) signaling pathway is essential for chronic inflammatory pain (Sanna and Galeotti, 2018). Inhibition of JNK signaling was effective in relieving inflammatory pain and NP in several animal models ( $\mathrm{Li}$ et al., 2017). The upregulation of Jun also showed protective and nerve-regenerative roles in DRG and Schwann cells in a nerve injury model (Yang et al., 2012; Zhao et al., 2014). JNK inhibitors may enhance the expression of activated transcription factor 3 and decrease the expression of multiple neurotrophic factors secreted by the DRG ( $\mathrm{Tu}$ et al., 2016). Thus, an increase in the expression of Jun may be correlated with post-CCD pain and nerve regeneration.

The role of $I L-6$ in nociceptive processing and regulation of pathological pain is well established (Schaper and RoseJohn, 2015). IL-6 was shown to induce the JAK-mediated phosphorylation of STAT proteins (Schaper and Rose-John, 2015) and participate in the later maintenance of NP by activating the JAK/STAT3 signaling pathway (Ding et al., 2016). Although the pain caused by $I L-6$ is subjectively unpleasant, several studies suggest that IL- 6 is beneficial to the nervous system. IL-6 is rapidly expressed in the retina and promotes the regeneration of the optic nerve by activating the JAK/STAT3 and PI3K/AKT signaling pathways following optic nerve injury (Leibinger et al., 2013). In general, $I L-6$ has detrimental and beneficial effects on the nervous system.

The upregulation of JAK/STAT3 signaling has been observed after nerve injury in animal models of neuropathic pain (Dominguez et al., 2008, 2010). Activation of the JAK/STAT3 cascade is induced by both pro-nociceptive (IL-6) and anti-nociceptive (IL-10) factors. These two forms of activation lead to the transcription of different pools of genes affecting the state of polarization of the microglia. The PI3K/AKT signaling pathway is also involved in the IL-6-mediated regulation of pathological pain (Ding et al., 2018). Plantar incisions have been found to induce time-dependent activation of the PI3K/Akt pathway in the spinal cord and DRG (Xu et al., 2014). As shown in Figure 4 and Table 2, the key candidate genes $J A K, I L-6$, Jun, and PI3K are vertices of a global signal transduction network and act synergistically; this is consistent with the findings of other studies. They are also involved in a complex physiological response that involves the termination of inflammation, promotion of the M2 state of microglial polarization, and synaptic plasticity (Nicolas et al., 2013; Xue et al., 2014).

\section{REFERENCES}

Attal, N., Lanteri-Minet, M., Laurent, B., Fermanian, J., and Bouhassira, D. (2011). The specific disease burden of neuropathic pain: results of a French nationwide survey. Pain 152, 2836-2843. doi: 10.1016/j.pain.2011.09.014

Bray, E. R., Yungher, B. J., Levay, K., Ribeiro, M., Dvoryanchikov, G., Ayupe, A. C., et al. (2019). Thrombospondin-1 mediates axon regeneration in retinal ganglion cells. Neuron 103, 642.e7-657.e7. doi: 10.1016/j.neuron.2019. 05.044
Our study has several limitations. First, tissue samples obtained from rats differ from those of humans and may not show the same target genes after nerve injury. Second, we detected the DEGs 7 days after CCD, which may reveal genes involved in the development, but not in the maintenance of NP. Third, the analysis of a larger number of tissue samples is required to improve the reliability of these results.

In conclusion, we found that $S d c 1, P i 3 k, J a k 2$, Jun, IL6 , and the signaling pathways cytokine-cytokine receptor interaction, neuroactive ligand-receptor interaction, toll-like receptor signaling pathway, and PI3K-Akt signaling pathway have key roles in both nerve regeneration and NP. These results may provide deeper insight into the understanding of the mechanism of NP after CCD, and help to identify promising therapeutic targets. Further studies are needed to confirm the role of cytokines and signaling pathways in the development of neuropathic pain, both individually and in cooperation.

\section{DATA AVAILABILITY STATEMENT}

The microarray data discussed in this article have been submitted to the NCBI Gene Expression Omnibus and can be accessed through the GSE accession number (GSE145222).

\section{ETHICS STATEMENT}

Procedures were approved by the Chinese Institutional Animal Care Committee of Shandong University.

\section{AUTHOR CONTRIBUTIONS}

$\mathrm{ZD}, \mathrm{SY}$, and XS performed the experiments and analyzed the data. YZ and LZ wrote the manuscript. XJ revised the manuscript and provided critical appraisal. SYu and YZ conceived the original idea and designed the experiments for this study. All authors read and approved the final manuscript.

\section{FUNDING}

This work was supported by the National Natural Science Foundation of China (Nos. 81101453 and 81472159 , both to YZ).

\section{ACKNOWLEDGMENTS}

We appreciate the technical assistance of the cardiovascular research center of Qilu Hospital.

Cobos, E. J., Nickerson, C. A., Gao, F., Chandran, V., Bravo-Caparros, I., GonzalezCano, R., et al. (2018). Mechanistic differences in neuropathic pain modalities revealed by correlating behavior with global expression profiling. Cell Rep. 22, 1301-1312. doi: 10.1016/j.celrep.2018.01.006

Couchman, J. R. (2003). Syndecans: proteoglycan regulators of cell-surface microdomains? Nat. Rev. Mol. Cell Biol. 4, 926-937. doi: 10.1038/ nrm 1257

Ding, C. P., Guo, Y. J., Li, H. N., Wang, J. Y., and Zeng, X. Y. (2018). Red nucleus interleukin-6 participates in the maintenance of neuropathic pain 
through JAK/STAT3 and ERK signaling pathways. Exp. Neurol. 300, 212-221. doi: 10.1016/j.expneurol.2017.11.012

Ding, C. P., Xue, Y. S., Yu, J., Guo, Y. J., Zeng, X. Y., and Wang, J. Y. (2016). The red nucleus interleukin-6 participates in the maintenance of neuropathic pain induced by spared nerve injury. Neurochem. Res. 41, 3042-3051. doi: 10.1007/s11064-016-2023-9

Dominguez, E., Mauborgne, A., Mallet, J., Desclaux, M., and Pohl, M. (2010). SOCS3-mediated blockade of JAK/STAT3 signaling pathway reveals its major contribution to spinal cord neuroinflammation and mechanical allodynia after peripheral nerve injury. J. Neurosci. 30, 5754-5766. doi: 10.1523/jneurosci. 5007-09.2010

Dominguez, E., Rivat, C., Pommier, B., Mauborgne, A., and Pohl, M. (2008). JAK/STAT3 pathway is activated in spinal cord microglia after peripheral nerve injury and contributes to neuropathic pain development in rat. J. Neurochem. 107, 50-60. doi: 10.1111/j.1471-4159.2008.05566.x

Du, Z., Song, X., Yan, F., Wang, J., Zhao, Y., and Liu, S. (2018). Genomewide transcriptional analysis of BRD4-regulated genes and pathways in human glioma U251 cells. Int. J. Oncol. 52, 1415-1426. doi: 10.3892/ijo.2018.4324

Edwards, T. J., and Hammarlund, M. (2014). Syndecan promotes axon regeneration by stabilizing growth cone migration. Cell Rep. 8, 272-283. doi: 10.1016/j.celrep.2014.06.008

Finnerup, N. B., Haroutounian, S., Kamerman, P., Baron, R., Bennett, D. L., Bouhassira, D., et al. (2016). Neuropathic pain: an updated grading system for research and clinical practice. Pain 157, 1599-1606. doi: 10.1097/j.pain. 0000000000000492

Gao, Y., Sun, N., Wang, L., Wu, Y., Ma, L., Hong, J., et al. (2018). Bioinformatics analysis identifies p53 as a candidate prognostic biomarker for neuropathic pain. Front. Genet. 9:320. doi: 10.3389/fgene.2018.00320

Gold, M. S., and Gebhart, G. F. (2010). Nociceptor sensitization in pain pathogenesis. Nat. Med. 16, 1248-1257. doi: 10.1038/nm.2235

Jacobs, D. S. (2017). Diagnosis and treatment of ocular pain: the ophthalmologist's perspective. Curr. Ophthalmol. Rep. 5, 271-275. doi: 10.1007/s40135-017$0152-1$

Jia, L., Zhang, Y., Qu, Y. J., Huai, J., Wei, H., and Yue, S. W. (2018). Gene therapy by lentivirus-mediated RNA interference targeting extracellular-regulated kinase alleviates neuropathic pain in vivo. J. Cell. Biochem. doi: 10.1002/jcb. 28090 [Epub ahead of print]

Kenney, A. M., and Kocsis, J. D. (1998). Peripheral axotomy induces long-term c-Jun amino-terminal kinase- 1 activation and activator protein- 1 binding activity by c-Jun and junD in adult rat dorsal root ganglia in vivo. J. Neurosci. $18,1318-1328$.

Leibinger, M., Andreadaki, A., Diekmann, H., and Fischer, D. (2013). Neuronal STAT3 activation is essential for CNTF- and inflammatory stimulationinduced CNS axon regeneration. Cell Death Dis. 4:e805. doi: 10.1038/cddis. 2013.310

Li, H., Li, Q., Xie, K., Feng, S., Wang, P., and Ma, X. (2013). Expression of c-Fos and c-Jun in adjacent cervical spinal cord segments following $\mathrm{C}_{7}$ nerve root rhizotomy in rats: Indication of a neural pathway between adjacent cervical spinal cord segments. Exp. Ther. Med. 6, 373-377. doi: 10.3892/etm.2013.1136

Li, J., Zhao, P. P., Hao, T., Wang, D., Wang, Y., Zhu, Y. Z., et al. (2017). Urotensin II inhibitor eases neuropathic pain by suppressing the JNK/NF-KB pathway. J. Endocrinol. 232, 165-174. doi: 10.1530/joe-16-0255

Liu, H., Xia, T., Xu, F., Ma, Z., and Gu, X. (2018). Identification of the key genes associated with neuropathic pain. Mol. Med. Rep. 17, 6371-6378. doi: 10.3892/mmr.2018.8718

Murakami, K., Tanaka, T., Bando, Y., and Yoshida, S. (2015). Nerve injury induces the expression of syndecan-1 heparan sulfate proteoglycan in primary sensory neurons. Neuroscience 300, 338-350. doi: 10.1016/j.neuroscience.2015. 05.033

Nicolas, C. S., Amici, M., Bortolotto, Z. A., Doherty, A., Csaba, Z., Fafouri, A., et al. (2013). The role of JAK-STAT signaling within the CNS. JAKSTAT 2:e22925. doi: $10.4161 /$ jkst.22925

Oh, S. B., Tran, P. B., Gillard, S. E., Hurley, R. W., Hammond, D. L., and Miller, R. J. (2001). Chemokines and glycoprotein 120 produce pain hypersensitivity by directly exciting primary nociceptive neurons. J. Neurosci. 21, 5027-5035. doi: 10.1523/jneurosci.21-14-05027.2001

Qu, Y. J., Zhang, X., Fan, Z. Z., Huai, J., Teng, Y. B., Zhang, Y., et al. (2016). Effect of TRPV4-p38 MAPK pathway on neuropathic pain in rats with chronic compression of the dorsal root ganglion. Biomed. Res. Int. 2016:6978923. doi: $10.1155 / 2016 / 6978923$

Rosenhouse-Dantsker, A. (2019). Cholesterol binding sites in inwardly rectifying potassium channels. Adv. Exp. Med. Biol. 1135, 119-138. doi: 10.1007/978-3030-14265-0_7

Sanna, M. D., and Galeotti, N. (2018). The HDAC1/c-JUN complex is essential in the promotion of nerve injury-induced neuropathic pain through JNK signaling. Eur. J. Pharmacol. 825, 99-106. doi: 10.1016/j.ejphar.2018.02.034

Schaper, F., and Rose-John, S. (2015). Interleukin-6: biology, signaling and strategies of blockade. Cytokine Growth Factor Rev. 26, 475-487. doi: 10.1016/j. cytogfr.2015.07.004

Tang, S., Jing, H., Huang, Z., Huang, T., Lin, S., Liao, M., et al. (2020). Identification of key candidate genes in neuropathic pain by integrated bioinformatic analysis. J. Cell. Biochem. 121, 1635-1648. doi: 10.1002/jcb.29398

Teixeira, M. J., da Paz, M. G., Bina, M. T., Santos, S. N., Raicher, I., Galhardoni, R., et al. (2015). Neuropathic pain after brachial plexus avulsion-central and peripheral mechanisms. BMC Neurol. 15:73. doi: 10.1186/s12883-015-0329-x

Tu, N. H., Katano, T., Matsumura, S., Pham, V. M., Muratani, T., Minami, T., et al. (2016). Role of c-Jun $\mathrm{N}$-terminal kinase in late nerve regeneration monitored by in vivo imaging of thy1-yellow fluorescent protein transgenic mice. Eur. J. Neurosci. 43, 548-560. doi: 10.1111/ejn.13139

van Hecke, O., Austin, S. K., Khan, R. A., Smith, B. H., and Torrance, N. (2014). Neuropathic pain in the general population: a systematic review of epidemiological studies. Pain 155, 654-662. doi: 10.1016/j.pain.2013.11.013

Wang, J., Ma, S. H., Tao, R., Xia, L. J., Liu, L., and Jiang, Y. H. (2017). Gene expression profile changes in rat dorsal horn after sciatic nerve injury. Neurol. Res. 39, 176-182. doi: 10.1080/01616412.2016.1273590

Woolf, C. J., and Mannion, R. J. (1999). Neuropathic pain: aetiology, symptoms, mechanisms, and management. Lancet 353, 1959-1964. doi: 10.1016/s01406736(99)01307-0

Xu, B., Guan, X. H., Yu, J. X., Lv, J., Zhang, H. X., Fu, Q. C., et al. (2014). Activation of spinal phosphatidylinositol 3-kinase/protein kinase B mediates pain behavior induced by plantar incision in mice. Exp. Neurol. 255, 71-82. doi: 10.1016/j.expneurol.2014.02.019

Xue, Z. J., Shen, L., Wang, Z. Y., Hui, S. Y., Huang, Y. G., and Ma, C. (2014). STAT3 inhibitor WP1066 as a novel therapeutic agent for bCCI neuropathic pain rats. Brain Res. 1583, 79-88. doi: 10.1016/j.brainres.2014.07.015

Yang, J. A., He, J. M., Lu, J. M., and Jie, L. J. (2018). Jun, Gal, Cd74, and C1qb as potential indicator for neuropathic pain. J. Cell. Biochem. 119, 4792-4798. doi: $10.1002 /$ jcb. 26673

Yang, D. P., Kim, J., Syed, N., Tung, Y. J., Bhaskaran, A., Mindos, T., et al. (2012). p38 MAPK activation promotes denervated Schwann cell phenotype and functions as a negative regulator of Schwann cell differentiation and myelination. J. Neurosci. 32, 7158-7168. doi: 10.1523/JNEUROSCI.581211.2012

Zhang, G., and Yang, P. (2017). Bioinformatics genes and pathway analysis for chronic neuropathic pain after spinal cord injury. Biomed. Res. Int. 2017:6423021. doi: 10.1155/2017/6423021

Zhao, H., Duan, L. J., Sun, Q. L., Gao, Y. S., Yang, Y. D., Tang, X. S., et al. (2020). Identification of key pathways and genes in L4 dorsal root ganglion (DRG) after sciatic nerve injury via microarray analysis. J. Invest. Surg. 33, 172-180. doi: 10.1080/08941939.2018.1452996

Zhao, L., Qu, W., Wu, Y., Ma, H., and Jiang, H. (2014). Dorsal root ganglionderived Schwann cells combined with poly(lactic-co-glycolic acid)/chitosan conduits for the repair of sciatic nerve defects in rats. Neural Regen. Res. 9, 1961-1967. doi: 10.4103/1673-5374.145374

Conflict of Interest: The authors declare that the research was conducted in the absence of any commercial or financial relationships that could be construed as a potential conflict of interest.

Copyright $\odot 2020$ Du, Yin, Song, Zhang, Yue, Jia and Zhang. This is an open-access article distributed under the terms of the Creative Commons Attribution License (CC BY). The use, distribution or reproduction in other forums is permitted, provided the original author(s) and the copyright owner(s) are credited and that the original publication in this journal is cited, in accordance with accepted academic practice. No use, distribution or reproduction is permitted which does not comply with these terms. 